\title{
P123 Beyond Diameters, Flow Hemodynamics Quantified by Magnetic Resonance Imaging to Help Characterizing Aneurysmal Aorta
}

\section{Sophia Houriez-Gombaud-Saintonge ${ }^{1,2,}$, Ariel Pascaner ${ }^{3}$, Gilles Soulat ${ }^{4}$, Umit Gencer ${ }^{5}$, Thomas Dietenbeck ${ }^{6}$,} Damian Craiem ${ }^{7}$, Emilie Bollache ${ }^{6}$, Yasmina Chenoune ${ }^{2}$, Elie Mousseaux ${ }^{4}$, Nadjia Kachenoura ${ }^{1}$

\author{
${ }^{1}$ Laboratoire d'Imagerie Biomédicale \\ ${ }^{2}$ ESME Research Lab \\ ${ }^{3} I M E T T Y B$ - Universidad Favaloro - CONICET, Buenos Aires, Argentina \\ ${ }^{4}$ Hopital Européen Georges Pompidou, Paris, France \\ ${ }^{5}$ Hôpital Européen Georges Pompidou, Paris, France \\ ${ }^{6}$ Laboratoire d'Imagerie Biomédicale, Paris, France \\ ${ }^{7} I M E T T Y B$ - Universidad Favaloro - CONICET, Buenos Aires, France
}

\begin{abstract}
Purpose: Referral to surgery in thoracic aortic aneurysms (TAA) is based on maximal diameter (Dmax) measured from imaging, which is known to have a high diagnosis failure rate. In addition to geometry, $4 \mathrm{D}$ flow MRI provides a comprehensive time-resolved flow imaging. Thus, our aim was to evaluate the ability of $4 \mathrm{D}$ flow MRI-derived quantitative flow indices to characterize TAA.

Methods: We studied 20 patients with TAA and tricuspid valve (TAVd, Dmax $=43 \pm 5 \mathrm{~mm}$, Age $=66 \pm 14$ years) and 56 healthy controls (YC: 30 subjects, Age $=36 \pm 9$ years $\leq 50$ years, OC: 26 subjects, Age $=65 \pm 9$ years $>50$ years). All underwent $4 \mathrm{D}$ flow MRI. After aortic segmentation, ascending aorta (AA) backward flow volume (VBF) was calculated in addition to maximal velocity jet angle and eccentricity (Ecc). Receiver operating characteristic analysis was performed to assess the ability of flow indices to characterize $\mathrm{AA}=$ dilation.

Results: While AA Dmax was 1.4-fold higher in TAVd than OC, VBF increased by 6.5 folds and Ecc and Angle varied by 1.3 to 1.7 folds between the two groups. Moreover, VBF changed by 12.7 folds between the aneurysmal AA as compared to TAVd descending aorta. Finally, VBF increased consistently with age in all controls and was able to detect AA dilation with a 0.98 accuracy.

Conclusion: AA backward flow quantified from 4D flow MRI outperformed the previously described indices such as flow eccentricity and angle in the characterization of thoracic aortic aneurysms.

(C) 2019 Association for Research into Arterial Structure and Physiology. Publishing services by Atlantis Press International B.V. This is an open access article distributed under the CC BY-NC 4.0 license (http://creativecommons.org/licenses/by-nc/4.0/).
\end{abstract}

